\title{
Ocular medicines in children: the regulatory situation related to clinical research
}

\author{
Filomena Fortinguerra*, Antonio Clavenna and Maurizio Bonati
}

\begin{abstract}
Background: Many ocular medications are prescribed for paediatric patients, but the evidence for their rational use is very scant. This study was planned to compare the availability and the licensing status of ocular medications marketed in Italy, the United Kingdom (UK), and the United States of America (USA) related to the amount of published and un-published RCTs testing these drugs in the paediatric population.

Methods: A quantitative analysis was performed to evaluate the number of ocular medications with a paediatric license in Italy, the UK, and the USA. A literature search was also performed in MEDLINE, EMBASE, and The Cochrane Central Register of Controlled Trials for randomized controlled trials (RCTs) on ophthalmic pharmacological therapy in children aged $<18$ years, published up to December 2010. A search in the international clinical trial registries, the list of paediatric investigation plans (PIPs) approved by European Medicines Agency (EMA), and the table of medicines with new paediatric information approved by Food and Drug Administration (FDA) was also performed.

Results: In all, of 197 drugs identified, 68 (35\%) single drugs are licensed for paediatric use at least in one considered country, while 23 (12\%) were marketed in all three countries. More specifically, in Italy 43 single drugs (48\% of those marketed) had a paediatric license, while 39 (64\%) did in the UK and 22 (54\%) did in the USA. Only 13 drugs were marketed with a paediatric license in all countries.

The percentage of drugs licensed for paediatric use and for which at least one RCT had been performed ranged between 51\% in Italy and 55\% in the USA. No published RCTs were found for 11 (48\%) drugs licensed for paediatric use in all three countries. In all, 74 (35\%) of the retrieved RCTs involved mydriatic/cycloplegic medications.

A total of 62 RCTs (56\% completed) on 46 drugs were found in the international clinical trial registries. Cyclosporin and bevacizumab were being studied in many ongoing trials. Twenty-six drugs had new paediatric information approved by FDA based on new paediatric clinical trials, while only 4 PIPs were approved by EMA.
\end{abstract}

Conclusions: There is a pressing need for further research and clinical development in the pediatric ophthalmic area, where effective up-to-date treatments, and additional research and education on use in children, remain priorities.

Keywords: review, ocular medicines, eye diseases, drug therapy, paediatrics

\section{Background}

Many drugs on the market labelled for adult use contain no information on paediatric use because their safety and efficacy have not been well studied in paediatric patients [1]. Many widely used drugs therefore include disclaimers stating that the paediatric use is "not recommended".

\footnotetext{
* Correspondence: filomena.fortinguerra@marionegri.it

Laboratory for Mother and Child Health, Department of Public Health, Mario Negri Institute for Pharmacological Research, Milan, Italy
}

Despite the prevalence of eye disease in early childhood (in the United Kingdom, by 3 years of age $5.7 \%$ of children had had $\geq 1$ eye condition, $0.24 \%$ of which associated with visual impairment) [2] more than in other paediatric areas, evidence for the rational use of ocular medicines in these patients is very scant.

Many ocular medications are used in children to treat common bacterial and viral infections, inflammation and allergy, uveitis and glaucoma, as well as other conditions

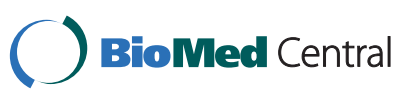


including myopia, amblyopia, and strabismus [3], even if data regarding their safety and effectiveness in the paediatric population are sparse. In 2000, a review of the 98 most commonly used or prescribed topical ophthalmic drugs found that only $51 \%$ provided information on paediatric use [4]. Without adequate paediatric labelling information, practitioners treating eye disease in children may be forced to prescribe ocular medications in an "off-label" manner, placing their paediatric patients at risk for serious adverse reactions $[5,6]$.

Children are not small adults. Statements regarding paediatric drug use must be age-specific to indicate for which group a drug has been studied: newborns, infants, pre-school children, school-age children, and adolescents. These groups differ not only in size and body weight but in physiology and metabolism as well [7]. Children, in particular infants and neonates who have thin eye membranes, may be particularly vulnerable to systemic effects of topical ophthalmic drugs as the doses used are often not weight-adjusted and are similar to doses used in adults. Systemic absorption may have a greater impact in children than in adults due to their lower body mass, altered metabolic capacity, and an immature blood brain barrier, leading to potentially higher plasma levels for a longer period of time and to a much greater risk of serious systemic side effects [8].

In addition to these differences, other characteristics unique to the paediatric population include the lack of commercially available dosage forms and concentrations appropriate for paediatric patients and the lack of published research on the pharmacokinetics and clinical use of new drugs [9]. The result is the high frequency of serious medication errors.

A study was planned to compare the availability and the licensing status of ocular medications marketed in Italy, the United Kingdom (UK), and the United States of America (USA) related to the amount of published and un-published RCTs testing these drugs in the paediatric population.

\section{Methods}

Ocular medications were identified and classified according to the International Anatomic-Therapeutical-Chemical classification system (ATC) [10] as S01: antibiotics, antivirals, anti-allergy drugs, non-steroidal anti-inflammatory drugs (NSAIDs), steroids, diagnostic agents, lubricants, glaucoma medications, local anaesthetics, and vascular endothelial growth factor inhibitors (anti-VEGF drugs) and combinations. A quantitative analysis was performed to record the number of ophthalmic drugs available on the market and those approved for paediatric use in Italy, the UK, and USA. Data on the licensing status of individual drugs were obtained by consulting national formularies: Italy's Repertorio Farmaceutico Italiano (Refi) [11], the UK's British National Formulary (BNF) [12], and the USA's Physicians' Desk Reference ${ }^{\mathbb{B}}$ $\left(\mathrm{PDR}^{\circledR}\right)$ [13].

In order to collect randomized controlled trials (RCTs) on safety and efficacy of ophthalmic drugs in the paediatric population, a bibliographic search for ophthalmological therapy in children aged up to 18 years in the MEDLINE (1967 - December 2010), EMBASE (1975 December 2010), and Cochrane Central Register of Controlled Trials (1967 - December 2010) databases was performed. The MeSH search terms and additional keywords used in the search strategy were: child/infant/ newborn/adolescent, ophthalmology, drug therapy, and randomized controlled trials, limiting the results to human. To make the search more complete, the terms were searched for both in the database dictionaries and through the free text search option that covered the articles' titles and abstracts. All the references retrieved were collected and analyzed using the software program Reference Manager, version 11 (Institute for Scientific Information, Berkeley, California). The titles and abstracts were screened independently by two reviewers (FF and $\mathrm{AC}$ ) to assess the relevance of the studies. Contrasting results were reviewed by a third person (MB).

We also searched for guidelines concerning paediatric ophthalmology management in MEDLINE and EMBASE, in the National Guidance Clearinghouse, National Library of Guidelines Specialist Library, National Institute for Clinical Excellence (NICE), Australian National Health and Medical Research Council, Canadian Medical Association InfoBase and New Zealand Guidelines Group databases, and on the American Academy of Pediatrics, Canadian Pediatric Society, and Royal College of Pediatrics websites.

In addition, a search for paediatric clinical trials on ocular medications in the World Health Organization's International Clinical Trials Registry Platform (ICTRP) [14], the ClinicalTrials.gov registry [15], and the International Standard Randomized Controlled Trial Number Register (ISRCTN) [16] was performed in order to find which of these drugs are under paediatric investigation. Furthermore, the list of paediatric investigation plans (PIPs) approved by EMA [17], the "List of the active substances included in the work-sharing procedure in accordance with Articles 45 and 46 of the European Paediatric Regulation [18], and the FDA's "Table of Medicines with new paediatric information", a list of drugs approved for paediatric use resulting from the paediatric clinical trials performed in response to paediatric legislative initiatives [19], and the updated priority list for studies into offpatent paediatric medicinal products [20], were also consulted in order to assess if there is a gap between research and clinical practice. 


\section{Results}

\section{Quantitative analysis}

A total of 197 ocular medications were reported in the 2010 ATC index, respectively, 88 (45\%), 63 (31\%), and 41 (21\%) of which were marketed in Italy, the UK, and the USA.

In all, 68 (35\%) single drugs are licensed for paediatric use in at least one considered country, while 23 drugs $(12 \%)$ were marketed in all three countries. More specifically, in Italy 43 single drugs (48\% of those marketed) had a paediatric license, while 39 (64\%) did in the UK and 22 (54\%) did in the USA. Only 13 drugs were marketed with a paediatric license in all the countries (Table 1 ). Only 3 licensed drugs appear in the World Health Organization (WHO) list of paediatric essential drugs. Tetracycline as $1 \%$ eye ointment and adrenaline as $2 \%$ eye drops, considered essential drugs for children, were not licensed for paediatric use in any country.

Fifteen single drugs and six combinations (mainly antiinfective, anti-inflammatory, and anti-allergy medications) were licensed for paediatric use only in Italy, while 16 single drugs and 8 combinations were licensed only in the UK (mainly local anaesthetics and lubricants), and 2 single drugs and 8 combinations only in the USA (mainly antiinfective medications). Almost all anti-allergy medications and combinations had a paediatric license in all three countries, while no local anaesthetics are licensed for paediatric use in Italy and USA and no NSAIDs are in the UK.

Wide differences were found in the age groups for which the drugs were licensed and only for 6 drugs the age range is the same or similar in all countries.

\section{Qualitative analysis Bibliographic search}

The bibliographic search produced 158 RCTs on 69 single drugs and combinations, involving a total of 18,816 children (Table 2). The percentage of drugs licensed for paediatric use with at least one RCT ranged between 51\% in Italy and 55\% in the USA. No published RCTs were found for 11 (48\%) ocular medications licensed for paediatric use in all three countries.

In all, 74 retrieved RCTs (35\%) regarded mydriatic/ cycloplegic medications, mainly antimuscarinic agents. In particular, 31 RCTs involving 3,530 children belonging to all age groups studied atropine as eye drops $1 \%$, a drug licensed for paediatric use only in the UK ( $\geq 3$ months). In addition, 3 studies were available on pirenzepine, a drug not licensed for paediatric use in any country.

Regarding the treatment of allergic conjunctivitis, 49 (23\%) RCTs on 11 drugs were found: 22 studies involved 6 anti-histamine agents (azelastine, bepotastine, emedastine, ketotifen, levocabastine, and olopatadine) tested in children $\geq 3$ years, and 9 RCTs involved 3 mast cell stabilizers, such as lodoxamide, cromoglicate, and nedocromil, in children $\geq 4$ years. Bepotastine is the only drug unlicensed for paediatric use in all considered countries.

A total of 43 RCTs (21\%) concerned 20 antibacterial agents and their combinations, 8 of them (40\%) not licensed for paediatric use in any country considered, such as the fluoroquinolone besifloxacin as eye suspension $2 \%$, tested in 3 RCTs in children older than one year. Among the six combinations studied 3 were licensed for paediatric use in the UK and 2 in the USA, while none in Italy. In addition, the anti-infective agent povidone-iodine, licensed for use in children older than 1 month, was studied only in Italy in 3 RCTs.

Among the medications commonly used in ophthalmic surgical procedures (e.g. strabismus surgery) there were 7 local anaesthetics (proparacaine, not licensed for paediatric use in any country was the drug most studied), 3 steroids (dexamethasone, fluorometholone, and rimexolone), and 3 NSAIDs (diclofenac, ketorolac, and flurbiprofen).

Ten RCTs regarded anti-glaucoma agents: 7 were on 3 beta-blockers, 2 on carbonic anhydrase inhibitors, and the last one on an acethylcholinesterase inhibitor, echothophate iodide. The most studied drug is timolol, a beta-bloker licensed for use in children older than 1 month only in Italy, as well as the carbonic anhydrase inhibitor dorzolamide.

Phenylephrine, the only decongestant agent studied, licensed for paediatric use in all countries considered, was involved in $11 \mathrm{RCTs}$, in which it was used in combination with a mydriatic/cyclopegic agent for eye examinations in children. In one RCT involving 10 neonates, phenylephrine was used alone.

\section{Guidelines}

Eight guidelines on pharmacological management of eye diseases in children were found: they addressed acute bacterial conjunctivitis [21], amblyopia [22,23], strabismus [24], glaucoma [25], retinopathy of prematurity (ROP) $[26,27]$, and prophylaxis of neonatal ophthalmia [28] (Table 3). Those concerning screening methods for diagnosing eye diseases in the paediatric population without drug use were not reported.

Five guidelines ( 2 regarding ROP, 2 regarding amblyopia, and 1 regarding strabismus) recommended drug use only for screening or post-surgical therapy, and not for the pharmacological management of the disease in childhood.

Almost all of the drugs listed in the guidelines are not licensed for use in children in any country considered, especially for prophylaxis of neonatal ophthalmia (no drug licensed), for the medical management of childhood glaucoma (5 out of 8 drugs are unlicensed) and acute bacterial conjunctivitis ( 8 out of 22 drugs are unlicensed). 
Table 1 Paediatric licensing status and number of RCTs related to ocular medications

\begin{tabular}{|c|c|c|c|c|c|c|}
\hline \multirow[t]{2}{*}{ Pharmaco-therapeutic Group } & \multirow[t]{2}{*}{ Drug name } & \multicolumn{3}{|c|}{ Licence Status } & \multicolumn{2}{|c|}{ RCTs } \\
\hline & & IT & UK & USA & Published & $\begin{array}{c}\text { Non- } \\
\text { published }\end{array}$ \\
\hline ANTI-ALLERGY MEDICATIONS & & $14 / 12^{*}$ & $8 / 8^{*}$ & $11 / 10^{*}$ & 34 & 5 \\
\hline \multirow[t]{6}{*}{ Anti-histamine agent } & Azelastine & $\geq 4$ yrs & $\geq 12 \mathrm{yrs}$ & $\geq 3 \mathrm{yrs}$ & 4 & - \\
\hline & Emedastine & $\geq 3$ yrs & $\geq 3 \mathrm{yrs}$ & $\geq 3 \mathrm{yrs}$ & 1 & - \\
\hline & Epinastine & $\geq 12 \mathrm{yrs}$ & $\geq 12$ yrs & $\geq 3 \mathrm{yrs}$ & - & - \\
\hline & Ketotifen & $\geq 3$ yrs & $\geq 3 \mathrm{yrs}$ & $\geq 3 \mathrm{yrs}$ & 5 & 3 \\
\hline & Levocabastine & all & $N A^{* *}$ & NA & 6 & - \\
\hline & Olopatadine & $\geq 3 \mathrm{yrs}$ & $\geq 3 \mathrm{yrs}$ & $\geq 3 \mathrm{yrs}$ & 4 & 1 \\
\hline \multirow[t]{5}{*}{ Mast cell stabilizer } & Spaglumic acid & all & NA & NA & - & - \\
\hline & Lodoxamide & all & $\geq 4$ yrs & $>2 \mathrm{yrs}$ & 1 & - \\
\hline & Nedocromil sodium & $\geq 6$ yrs & $\geq 6 \mathrm{yrs}$ & $\geq 3 \mathrm{yrs}$ & 5 & - \\
\hline & Sodium cromogligate & $n s^{* *}$ & all & NA & 6 & - \\
\hline & Pemirolast & NA & NA & $\geq 3 \mathrm{yrs}$ & - & - \\
\hline \multirow[t]{3}{*}{ Decongestant (Sympathomimetic agent) } & Naphazoline & $\geq 10 \mathrm{yrs}$ & NA & $\mathrm{nl}$ & - & 1 \\
\hline & Oxymetazoline & $\geq 3$ yrs & NA & $\geq 6 \mathrm{yrs}$ & - & - \\
\hline & Tetryzoline & $\geq 3$ yrs & NA & $\geq 6 \mathrm{yrs}$ & - & - \\
\hline ANTI-ALLERGY COMBINATIONS & & $3 / 3$ & $1 / 1$ & $2 / 2$ & 0 & 0 \\
\hline \multirow[t]{3}{*}{ Anti-histamine agent + Decongestant } & Antazoline + Xylometazoline & NA & $\geq 5$ yrs & NA & - & - \\
\hline & $\begin{array}{l}\text { Chlorpheniramine + } \\
\text { Tetryzoline }\end{array}$ & $\geq 3$ yrs & NA & NA & - & - \\
\hline & Pheniramine + Tetryzoline & $\geq 3$ yrs & NA & NA & - & - \\
\hline Mast cell stabilizer + Decongestant & Cromoglicate + Tetryzoline & $\geq 3$ yrs & NA & NA & - & - \\
\hline Astringent + Decogestant & Zinc sulfate + Tetryzoline & NA & NA & $\geq 6 \mathrm{yrs}$ & - & - \\
\hline Decogestant + Lubricants & $\begin{array}{l}\text { Tetryzoline + Povidone + } \\
\text { Dextran } 70+\text { Polyethylene } \\
\text { glycol } 400\end{array}$ & NA & NA & $\geq 6 \mathrm{yrs}$ & - & - \\
\hline ANTI-GLAUCOMA MEDICATIONS & & $23 / 6$ & $14 / 1$ & $6 / 1$ & 4 & 3 \\
\hline Beta-blocker & Timolol & $>1 \mathrm{~m}$ & $n l^{* *}$ & $\mathrm{nl}$ & 4 & 2 \\
\hline Carbonic anhydrase inhibitor & Dorzolamide & all & $\mathrm{nl}$ & ns & - & \\
\hline \multirow{3}{*}{$\begin{array}{l}\text { Sympathomimetic agent (selective } \alpha_{2}- \\
\text { agonist) }\end{array}$} & Apraclonidine & $\geq 12 \mathrm{yrs}$ & $\geq 12 \mathrm{yrs}$ & $\mathrm{nl}$ & - & - \\
\hline & Brimonidine & $\geq 12 \mathrm{yrs}$ & NA & $\geq 2 \mathrm{yrs}$ & - & 1 \\
\hline & Clonidine & ns & NA & NA & - & - \\
\hline \multirow[t]{2}{*}{ Parasympathomimetic (colinergic) agent } & Aceclidine & $\geq 3$ yrs & NA & NA & - & - \\
\hline & Pilocarpine & $\geq 3$ yrs & $\mathrm{nl}$ & $\mathrm{Nl}$ & - & - \\
\hline ANTI-GLAUCOMA COMBINATIONS & & $2 / 1$ & $1 / 0$ & $2 / 2$ & 1 & 0 \\
\hline Beta-blocker + Carbonic anhydrase inhibitor & Timolol + Dorzolamide & $\geq 2$ yrs & $\mathrm{nl}$ & $\geq 2 \mathrm{yrs}$ & 1 & - \\
\hline Beta-blocker + Sympathomimetic agent & Timolol + Brimonidine & $\mathrm{nl}$ & NA & $\geq 2 \mathrm{yrs}$ & - & - \\
\hline ANTI-INFLAMMATORY MEDICATIONS & & $16 / 8$ & $8 / 5$ & $5 / 2$ & 16 & 6 \\
\hline \multirow{3}{*}{$\begin{array}{l}\text { Non-Steroidal Anti-Inflammatory Drug } \\
\text { (NSAID) }\end{array}$} & Diclofenac & $\geq 3$ yrs & $\mathrm{nl}$ & $\mathrm{nl}$ & 4 & 1 \\
\hline & Indomethacin & $\geq 3 \mathrm{yrs}$ & NA & NA & - & - \\
\hline & Ketorolac & NA & $\mathrm{nl}$ & $\geq 3 \mathrm{yrs}$ & 3 & - \\
\hline \multirow[t]{4}{*}{ Steroid agent } & Betamethasone & NA & all & NA & - & - \\
\hline & Desonide & $>1 \mathrm{~m}$ & NA & NA & - & - \\
\hline & Dexamethasone & $>1 \mathrm{~m}$ & all & $\mathrm{nl}$ & 6 & 2 \\
\hline & Fluorometholone & $\geq 2 \mathrm{yrs}$ & $\geq 2 \mathrm{yrs}$ & $\geq 2 \mathrm{yrs}$ & 3 & 1 \\
\hline
\end{tabular}


Table 1 Paediatric licensing status and number of RCTs related to ocular medications (Continued)

\begin{tabular}{|c|c|c|c|c|c|c|}
\hline & Hydrocortisone & $>1 \mathrm{~m}$ & all & NA & - & - \\
\hline & Prednisolone & NA & all & $\mathrm{nl}$ & - & 2 \\
\hline & Clobetasone & $>1 \mathrm{~m}$ & NA & NA & - & - \\
\hline \multirow[t]{2}{*}{ Steroid agent + Decongestant } & $\begin{array}{l}\text { Fluorometholone + } \\
\text { Tetryzoline }\end{array}$ & $\geq 2$ yrs & NA & NA & - & - \\
\hline & Clobetasone + Tetryzoline & ns & NA & NA & - & - \\
\hline ANTI-INFECTIVE MEDICATIONS & & $21 / 13$ & $9 / 9$ & $10 / 7$ & 27 & 3 \\
\hline \multirow[t]{3}{*}{ Antibacterial agent } & Chloramphenicol & $\geq 3$ yrs & all & NA & 7 & - \\
\hline & Fusidic acid & ns & all & NA & 3 & - \\
\hline & Propamidine isetionate & NA & all & NA & - & - \\
\hline \multirow[t]{4}{*}{ Aminoglycoside } & Gentamycin & $\geq 3$ yrs & all & $>1 \mathrm{yr}$ & 2 & 1 \\
\hline & Neomycin & NA & all & NA & - & - \\
\hline & Netilmycin & $>1 \mathrm{~m}$ & NA & NA & - & - \\
\hline & Tobramycin & $\geq 1 \mathrm{yr}$ & NA & $\mathrm{nl}$ & 3 & - \\
\hline \multirow[t]{6}{*}{ Quinolone } & Ciprofloxacin & all & $\geq 1 \mathrm{yr}$ & $\geq 1 \mathrm{yr}$ & 2 & - \\
\hline & Gatifloxacin & NA & NA & $\geq 1 \mathrm{yr}$ & - & \\
\hline & Levofloxacin & $\geq 1 \mathrm{yr}$ & $\geq 1 \mathrm{yr}$ & $\geq 1 \mathrm{yr}$ & 2 & - \\
\hline & Lomefloxacine & $\geq 1 \mathrm{yr}$ & NA & NA & - & - \\
\hline & Moxifloxacin & $\geq 1 \mathrm{~m}$ & $\mathrm{nl}$ & $\geq 1 \mathrm{yr}$ & 3 & \\
\hline & Ofloxacin & ophtalmia & neonatorum & $>1 \mathrm{~m}$ & $\geq 1 \mathrm{yr}$ & 1 \\
\hline \multirow[t]{3}{*}{ Antiviral agent } & Acyclovir & all & all & $\mathrm{nl}$ & - & - \\
\hline & Idoxuridine & $\geq 3$ yrs & NA & NA & - & - \\
\hline & Trifluridine & all & NA & $\geq 6$ yrs & - & - \\
\hline Other anti-infective agent & Povidone - lodine & $>1 \mathrm{~m}$ & NA & $\mathrm{nl}$ & 4 & 2 \\
\hline ANTIBACTERIAL COMBINATIONS & & $9 / 2$ & $6 / 3$ & $4 / 1$ & 3 & 0 \\
\hline \multirow[t]{4}{*}{ Antibacterials } & Polimyxin B + Trimethoprim & NA & all & $>2 \mathrm{~ms}$ & 1 & - \\
\hline & Polimyxin B + Bacitracin & NA & all & $\mathrm{nl}$ & 1 & - \\
\hline & $\begin{array}{l}\text { Neomycin + Polymyxin B + } \\
\text { Gramicidin }\end{array}$ & NA & $\geq 2$ yrs & $\mathrm{nl}$ & - & - \\
\hline & $\begin{array}{l}\text { Neomycin + } \\
\text { Chloramphenicol }\end{array}$ & ns & NA & NA & - & - \\
\hline \multirow[t]{9}{*}{ Antibacterial + Steroid } & $\begin{array}{l}\text { Neomycin + Polymyxin B + } \\
\text { Dexamethasone }\end{array}$ & $\mathrm{nl}$ & all & $\geq 2$ yrs & - & - \\
\hline & $\begin{array}{l}\text { Neomycin + Polymyxin B + } \\
\text { Hydrocortisone }\end{array}$ & NA & NA & ns & - & - \\
\hline & $\begin{array}{l}\text { Neomycin }+ \\
\text { Chloramphenicol }+ \\
\text { Hydrocortisone }\end{array}$ & ns & NA & NA & - & - \\
\hline & Neomycin + Prednisolone & ns & all & NA & - & - \\
\hline & Neomycin + Fluocinolone & ns & NA & NA & - & - \\
\hline & Neomycin + Betamethasone & NA & all & NA & 1 & - \\
\hline & $\begin{array}{l}\text { Tobramycin + } \\
\text { Dexamethasone } \\
\end{array}$ & $\mathrm{nl}$ & NA & $\geq 2$ yrs & - & - \\
\hline & $\begin{array}{l}\text { Prednisolone + } \\
\text { Sulphacetamide } \\
\end{array}$ & NA & NA & $\geq 6$ yrs & - & - \\
\hline & $\begin{array}{l}\text { Tobramycin + } \\
\text { Fluorometholone }\end{array}$ & NA & NA & $\geq 2$ yrs & - & - \\
\hline Antibacterial + Steroid + Decongestant & $\begin{array}{l}\text { Neomycin + Gramicidin + } \\
\text { Tetryzoline }+ \\
\text { Dexamethasone }\end{array}$ & $\geq 3$ yrs & NA & NA & - & - \\
\hline
\end{tabular}


Table 1 Paediatric licensing status and number of RCTs related to ocular medications (Continued)

\begin{tabular}{|c|c|c|c|c|c|c|}
\hline & $\begin{array}{l}\text { Betamethasone }+ \\
\text { Sulphacetamide }+ \text { Tetryzoline }\end{array}$ & $\geq 2$ yrs & NA & NA & - & - \\
\hline MYDRIATIC/CYCLOPLEGIC MEDICATIONS & & $7 / 4$ & $6 / 5$ & $2 / 1$ & 55 & 3 \\
\hline \multirow[t]{5}{*}{ Antimuscarinic agent } & Cyclopentolate & $\geq 3$ yrs & $>3 \mathrm{~ms}$ & all & 14 & - \\
\hline & Homatropine & ns & $>3 \mathrm{~ms}$ & $\mathrm{nl}$ & - & - \\
\hline & Tropicamide & $>1 \mathrm{~m}$ & all & NA & 10 & - \\
\hline & Atropine & ns & $\begin{array}{c}>3 \mathrm{~ms}(\mathrm{nl} \\
\text { uveitis) }\end{array}$ & NA & 31 & 3 \\
\hline & Ibopamine & all & NA & NA & - & - \\
\hline Decongestant (Sympathomimetic agent) & Phenylephrine & $\geq 12$ yrs & $\begin{array}{l}\text { All (nl 10\% } \\
\text { drops) }\end{array}$ & NA & 2 & - \\
\hline PERI-OPERATIVE MEDICATIONS & & $2 / 0$ & $4 / 4$ & $1 / 0$ & 4 & 0 \\
\hline \multirow[t]{4}{*}{ Local anaesthetic } & Lidocaine & $\mathrm{nl}$ & all & $\mathrm{nl}$ & 2 & - \\
\hline & Oxybuprocaine & ns & all & NA & 1 & - \\
\hline & Proxymetacaine & NA & all & NA & - & - \\
\hline & Tetracaine & NA & all & NA & 1 & - \\
\hline LUBRICANTS AND ASTRIGENTS & & $5 / 0$ & $10 / 5$ & $4 / 0$ & 0 & 0 \\
\hline \multirow[t]{6}{*}{ Ocular lubricant and astringent } & Polyvinyl alcohol & Ns & all & NA & - & - \\
\hline & Carmellose sodium & ns & all & NA & - & - \\
\hline & Hydroxyethylcellulose & NA & all & NA & - & - \\
\hline & Paraffin & NA & all & NA & - & - \\
\hline & Sodium hyaluronate & ns & all & NA & - & - \\
\hline & Hypromellose & $\mathrm{NA}$ & all & $\mathrm{nl}$ & - & - \\
\hline LUBRICANT COMBINATIONS & & $0 / 0$ & $2 / 2$ & $6 / 2$ & 0 & 0 \\
\hline \multirow[t]{3}{*}{ Lubricants } & Hypromellose + Glycerin & NA & NA & all & - & - \\
\hline & Hypromellose + Dextran 70 & NA & all & $\mathrm{nl}$ & - & - \\
\hline & $\begin{array}{l}\text { Hypromellose + Glycerin + } \\
\text { Polyethylene glycol } 400\end{array}$ & NA & NA & $\geq 6$ yrs & - & - \\
\hline Lubricant + Steroid agent & $\begin{array}{l}\text { Hypromellose }+ \\
\text { Dexamethasone }\end{array}$ & NA & all & NA & - & - \\
\hline OTHER OCULAR MEDICATIONS & & $2 / 1$ & $2 / 2$ & $2 / 1$ & 0 & 0 \\
\hline Hypertonic agent & Sodium chloride & NA & all & $\mathrm{NI}$ & - & - \\
\hline Ocular diagnostic agent & Fluorescein & ns & all & $\mathrm{NA}$ & - & - \\
\hline Topical immunomodulator & Cyclosporine $0.05 \%$ & NA & NA & $\begin{array}{l}\geq 16 \\
\text { yrs }\end{array}$ & - & - \\
\hline Other ocular agent & Heparin & $>1 \mathrm{~m}$ & NA & $\mathrm{NA}$ & - & - \\
\hline TOTAL SINGLE DRUGS & 68 & $88 / 43$ & $61 / 39$ & $41 / 22$ & 140 & 20 \\
\hline TOTAL COMBINATIONS & 29 & $16 / 7$ & $10 / 6$ & $14 / 7$ & 4 & - \\
\hline
\end{tabular}

NOTE: Only drugs with a paediatric licence at least in one country are listed. The drugs in bold are listed in the WHO model list of essential medicines for children.

${ }^{*} \mathrm{~N}^{\circ}$ drugs marketed $/ \mathrm{N}^{\circ}$ drugs marketed with paediatric licence

** ns: not specified; nl: not licensed for paediatric use; NA: not authorised

The authors indicated that all these drugs are generally used in a off label manner and that the majority of data on these medications are from adult studies.

Finally, no guidelines on the pharmacological treatment of allergic conjunctivitis were found.

\section{Search for the paediatric RCTs in registries}

A search performed in the World Health Organization's International Clinical Trials Registry Platform (ICTRP), the ClinicalTrials.gov registry, and the International Standard Randomized Controlled Trial Number Register (ISRCTN) found 46 ocular medications currently under paediatric investigation in 62 RCTs (56\% of which completed). Cyclosporin, an immunosuppressant agent, and bevacizumab, a humanized monoclonal antibody, were the drugs involved in the most studies: 7 RCTs testing cyclosporine in the treatment of keratoconjunctivitis (4), 
Table 2 Summary of retrieved RCTs on the use of ocular medications in the paediatric population

\begin{tabular}{|c|c|c|c|c|c|}
\hline Pharmaco-therapeutic Group & Drug name & Formulation & $\mathrm{N}^{\circ} \mathrm{RCTs}$ & $\begin{array}{l}\mathrm{N}^{\circ} \\
\text { Children }\end{array}$ & $\begin{array}{l}\text { Age } \\
\text { range }\end{array}$ \\
\hline MYDRIATIC/CYCLOPLEGIC MEDICATIONS & & & $\begin{array}{l}74 \\
(35 \%)\end{array}$ & & \\
\hline \multirow[t]{6}{*}{ Antimuscarinic agent } & Atropine & eye drops $1 \%$ & 31 & 3530 & all \\
\hline & Cyclopentolate & eye drops $0.5 \%$ & 2 & 28 & $\leq 13 \mathrm{yrs}$ \\
\hline & & eye drops $1 \%$ & 11 & 181 & $\leq 16 \mathrm{yrs}$ \\
\hline & Tropicamide & eye drops $1 \%$ & 9 & 348 & all \\
\hline & Pirenzepine & ophthalmic gel 1\% & 3 & 276 & $6-12 \mathrm{yrs}$ \\
\hline & Cyclopentolate/Tropicamide & eye drops $1 \% / 1 \%$ & 6 & 176 & all \\
\hline Sympathomimetic agent & Phenylephrine & eye drops $2.5 \%$ & 1 & 10 & $\leq 1 \mathrm{~m}$ \\
\hline \multirow{5}{*}{$\begin{array}{l}\text { Antimuscarinic agent + Sympathomimetic } \\
\text { agent }\end{array}$} & Tropicamide/Phenylephrine & eye drops 1\%/2.5\% & 3 & 92 & $\leq 1 \mathrm{~m}$ \\
\hline & & eye drops $0.5 \% / 2.5 \%$ & 2 & 51 & $\leq 8 \mathrm{yrs}$ \\
\hline & & eye drops $0.5 \% / 0.5 \%$ & 1 & 12 & 3-11 yrs \\
\hline & $\begin{array}{l}\text { Cyclopentolate/ } \\
\text { Phenylephrine }\end{array}$ & eye drops 1\%/2.5\% & 2 & 30 & $\leq 6$ yrs \\
\hline & & eye drops $0.2 \% / 1 \%$ & 3 & 99 & $\leq 1 \mathrm{~m}$ \\
\hline ANTI-INFECTIVE MEDICATIO NS & & & $\begin{array}{l}51 \\
(24 \%)\end{array}$ & & \\
\hline \multirow[t]{17}{*}{ Antibacterial agent } & Chloramphenicol & eye drops $0.5 \%$ & 7 & 1664 & $\leq 12$ yrs \\
\hline & Azithromycin & eye drops $1 \%$ & 1 & 335 & $\geq 1 \mathrm{yr}$ \\
\hline & & eye drops $1.5 \%$ & 2 & 542 & $\geq 1 \mathrm{yr}$ \\
\hline & Tetracycline & eye drops $1 \%$ & 2 & 518 & $1-10$ yrs \\
\hline & & eye ointment $1 \%$ & 2 & 218 & $\geq 6 \mathrm{~ms}$ \\
\hline & Besifloxacin & eye suspension $0.6 \%$ & 3 & 1124 & $\geq 1 \mathrm{yr}$ \\
\hline & Fusidic acid & eye drops $1 \%$ & 3 & 594 & $\leq 2$ yrs \\
\hline & Moxifloxacin & eye drops $0.5 \%$ & 3 & 645 & all \\
\hline & Tobramycin & eye drops & 3 & 741 & $\leq 12$ yrs \\
\hline & Ciprofloxacin & eye drops $0.3 \%$ & 2 & 193 & $\leq 12 \mathrm{yrs}$ \\
\hline & $\underline{\text { Levofloxacin }}$ & eye drops $0.5 \%$ & 2 & 106 & $1-16$ yrs \\
\hline & Gentamycin & eye ointment & 2 & 117 & $\leq 12$ yrs \\
\hline & Erythromycin & eye drops & 1 & 110 & $\leq 1 \mathrm{~m}$ \\
\hline & & eye ointment & 1 & 24 & $\leq 1 \mathrm{yr}$ \\
\hline & Ofloxacin & eye drops $0.3 \%$ & 1 & 23 & $\geq 2$ yrs \\
\hline & Oxytetracycline & eye drops $1 \%$ & 1 & 450 & $\leq 1 \mathrm{~m}$ \\
\hline & Sulphacetamide & eye drops $10 \%$ & 1 & 14 & $\leq 1 \mathrm{~m}$ \\
\hline \multirow[t]{3}{*}{ Antibacterials combinations } & Polymixin B/Oxytetracycline & eye ointment & 2 & 132 & $2-10$ yrs \\
\hline & Polymixin B/Bacitracin & eye ointment & 1 & 66 & $\geq 1 \mathrm{~m}$ \\
\hline & Polymixin B/Trimethoprim & eye drops & 1 & 28 & all \\
\hline Antibacterial agent + NSAID & Gentamycin/Diclofenac & eye drops & 1 & 12 & $\leq 12 \mathrm{yrs}$ \\
\hline \multirow[t]{2}{*}{ Antibacterial agent + Steroid agent } & Neomycin/Betamethasone & eye drops & 1 & 12 & $\leq 12 \mathrm{yrs}$ \\
\hline & Tobramycin/Dexamethasone & eye drops & 1 & 28 & $4-10$ yrs \\
\hline \multirow[t]{2}{*}{ Antifungal agents } & Miconazole & eye suspension 1\% & 1 & 12 & $\geq 15 \mathrm{yr}$ \\
\hline & Econazole/Miconazole & eye suspension $1 \% / 1 \%$ & 1 & 7 & $\geq 15 \mathrm{yr}$ \\
\hline \multirow[t]{2}{*}{ Other anti-infective eye preparation } & Povidone-iodine & eye drops $2.5 \%$ & 4 & 3132 & $\leq 1 \mathrm{yr}$ \\
\hline & Silver nitrate & eye drops 1\% & 1 & 450 & $\leq 1 \mathrm{~m}$ \\
\hline ANTI-ALLERGY MEDICATIONS & & & $\begin{array}{l}31 \\
(15 \%)\end{array}$ & & \\
\hline
\end{tabular}


Table 2 Summary of retrieved RCTs on the use of ocular medications in the paediatric population (Continued)

\begin{tabular}{|c|c|c|c|c|c|}
\hline \multirow[t]{7}{*}{ Anti-histamine agent } & Levocabastine & eye suspension $0.5 \%$ & 6 & 174 & $\geq 3 \mathrm{yrs}$ \\
\hline & Ketotifen & eye drops $0.025 \%$ & 5 & 522 & $\geq 3 \mathrm{yrs}$ \\
\hline & Olopatadine & eye drops $0.2 \%$ & 4 & 99 & $\geq 3 \mathrm{yrs}$ \\
\hline & Azelastine & eye drops $0.02 \%$ & 4 & 132 & $\geq 4 \mathrm{yrs}$ \\
\hline & Bepotastine & eye drops $1 \%$ & 1 & 36 & $\geq 10 \mathrm{yrs}$ \\
\hline & & eye drops $1.5 \%$ & 1 & 36 & $\geq 10 \mathrm{yrs}$ \\
\hline & Emedastine & eye drops $0.05 \%$ & 1 & - & $3-16$ yrs \\
\hline \multirow[t]{4}{*}{ Mast cell stabilizer } & Lodoxamide & eye drops $0.1 \%$ & 1 & 15 & $\geq 6$ yrs \\
\hline & Cromoglycate & eye drops $2 \%$ & 4 & 128 & $\geq 4 \mathrm{yrs}$ \\
\hline & & eye drops $4 \%$ & 1 & 30 & $\geq 16$ yrs \\
\hline & Nedocromil & eye drops $2 \%$ & 3 & 85 & $\geq 4 \mathrm{yrs}$ \\
\hline \multicolumn{2}{|l|}{ ANTI-INFLAMMATORY MEDICATIONS } & & \multicolumn{2}{|l|}{$18(9 \%)$} & \\
\hline \multirow[t]{3}{*}{ Corticosteroid } & Dexamethasone & eye drops $0.1 \%$ & 6 & 159 & all \\
\hline & Fluorometholone & eye drops $0.1 \%$ & 3 & 52 & $\leq 10 \mathrm{yrs}$ \\
\hline & Rimexolone & eye drops $1 \%$ & 1 & 22 & $4-8$ yrs \\
\hline \multirow[t]{3}{*}{ NSAID } & Diclofenac & eye drops $0.1 \%$ & 4 & 93 & $\geq 2 \mathrm{yrs}$ \\
\hline & Ketorolac & eye drops $0.5 \%$ & 3 & 70 & $\leq 12 \mathrm{yrs}$ \\
\hline & Flurbiprofen & eye drops & 1 & 50 & $\geq 5 \mathrm{yrs}$ \\
\hline \multicolumn{2}{|l|}{ ANTIGLAUCOMA MEDICATIONS } & & \multicolumn{2}{|l|}{$10(5 \%)$} & \\
\hline \multirow[t]{6}{*}{ Beta-blocker } & Timolol & eye drops $0.25 \%$ & 2 & 44 & $7-13$ yrs \\
\hline & & eye drops $0.5 \%$ & 1 & 12 & $\geq 14$ yrs \\
\hline & & $\begin{array}{l}\text { gel-forming solution } \\
0.25 \%\end{array}$ & 1 & 35 & $\leq 6 y r s$ \\
\hline & & gel-forming solution $0.5 \%$ & 1 & 36 & $\leq 6 y r s$ \\
\hline & Betaxolol & eye suspension $0.25 \%$ & 1 & 52 & $\leq 6 \mathrm{yrs}$ \\
\hline & Levobetaxolol & eye suspension $0.5 \%$ & 1 & 46 & $\leq 6 \mathrm{yrs}$ \\
\hline \multirow[t]{2}{*}{ Carbonic anhydrase inhibitor } & Brinzolamide & eye suspension $1 \%$ & 1 & 32 & $\leq 6$ yrs \\
\hline & Dorzolamide & eye drops $2 \%$ & 1 & 56 & $\leq 6 \mathrm{yrs}$ \\
\hline Acetylcholinesterase inhibitor & Echothophate iodide & eye drops & 1 & 20 & - \\
\hline \multicolumn{2}{|l|}{ PERI-OPERATIVE MEDICATIONS } & & \multicolumn{2}{|l|}{$18(9 \%)$} & \\
\hline \multirow[t]{10}{*}{ Local anaesthetic agent } & Bupivacaine & subconjuntival infiltration & 2 & 38 & $5-10$ yrs \\
\hline & & eye drops $0.5 \%$ & 1 & 17 & $3-6$ yrs \\
\hline & Proparacaine & eye drops $0.5 \%$ & 3 & 58 & $\leq 1 \mathrm{~m}$ \\
\hline & Lidocaine & eye drops $2 \%$ & 1 & 10 & $3-14$ yrs \\
\hline & & ophthalmic gel $2 \%$ & 1 & 24 & $3-12$ yrs \\
\hline & Amethocaine & eye drops $0.5 \%$ & 2 & 45 & $2-8$ yrs \\
\hline & Levobupivacaine & eye drops & 1 & 13 & $1-16$ yrs \\
\hline & Oxybuprocaine & eye drops $0.4 \%$ & 1 & 20 & $3-8$ yrs \\
\hline & Tetracaine & eye drops $1 \%$ & 1 & 44 & $1-12$ yrs \\
\hline & Sucrose & eye drops & 1 & 11 & $\leq 1 \mathrm{~m}$ \\
\hline \multirow[t]{4}{*}{ Chemotherapeutic agents } & Mitomicyn C & eye drops $0.02 \%$ & 1 & 10 & $\geq 6$ yrs \\
\hline & & ocular injection $0.4 \%$ & 1 & 7 & $\geq 6$ yrs \\
\hline & 5-fluorouracil & ocular injection & 1 & 4 & $\leq 12 \mathrm{yrs}$ \\
\hline & Mitomicyn C/5-fluorouracil & ocular injection & 1 & 4 & $\leq 12 \mathrm{yrs}$ \\
\hline \multicolumn{2}{|l|}{ OTHER DRUGS } & & \multicolumn{2}{|l|}{$7(3 \%)$} & \\
\hline \multirow[t]{3}{*}{ Vernal keratoconjunctivitis } & Cyclosporine & eye drops $2 \%$ & 1 & 14 & $5-16$ yrs \\
\hline & & eye drops $1.25 \%$ & 1 & 20 & 5-14 yrs \\
\hline & & eye drops $1 \%$ & 1 & 32 & 5-14 yrs \\
\hline
\end{tabular}


Table 2 Summary of retrieved RCTs on the use of ocular medications in the paediatric population (Continued)

\begin{tabular}{|c|c|c|c|c|c|}
\hline & Mipragoside & ophthalmic gel $0.5 \%$ & 1 & 12 & $5-20$ yrs \\
\hline ROP therapy & Bevacizumab & intravitreal injection & 1 & 7 & $\leq 1 \mathrm{~m}$ \\
\hline Esotropia & Botulinum toxin & ocular injection & 1 & 27 & $6-12$ yrs \\
\hline Dacryocystitis & Herba houttuyaniae & eye drops & 1 & 268 & $\leq 1 \mathrm{~m}$ \\
\hline TOTAL (69 single drugs \& combinations) & & & 209 & 18,816 & \\
\hline
\end{tabular}

NOTE: the total is higher than the sum of the RCTs (158) because some drugs were tested in more than one trial. The references to RCTs are available upon request to the corresponding author.

Table 3 Summary of guidelines on pharmacological therapy of ocular disease in the paediatric population

\begin{tabular}{|c|c|c|c|c|c|c|c|}
\hline Ref. & Organisation & Title & Disease & $\begin{array}{l}\text { Quality } \\
\text { of } \\
\text { evidence }\end{array}$ & Treatment (Licensing status) & Country & Year \\
\hline$\overline{[41]}$ & $\begin{array}{l}\text { National Guideline } \\
\text { Clearinghause (NGC) }\end{array}$ & $\begin{array}{l}\text { Guidelines for the } \\
\text { treatment and } \\
\text { management of acute } \\
\text { bacterial conjunctivitis in } \\
\text { children and adults. }\end{array}$ & $\begin{array}{l}\text { Acute bacterial } \\
\text { conjunctivitis }\end{array}$ & 1 & 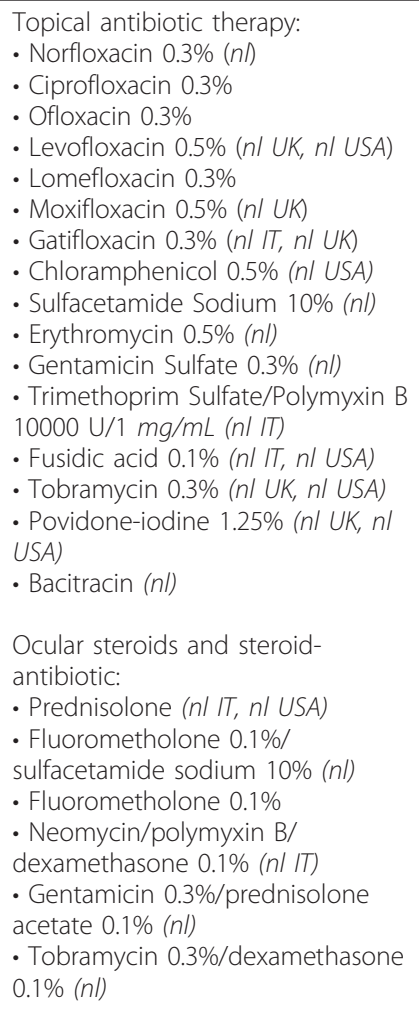 & USA & 2005 \\
\hline [34] & Canadian Paediatric Society & $\begin{array}{l}\text { Recommendations for } \\
\text { the prevention of } \\
\text { neonatal ophthalmia }\end{array}$ & $\begin{array}{l}\text { Prophylaxis to } \\
\text { prevent } \\
\text { neonatal } \\
\text { ophthalmia due } \\
\text { to } \mathrm{N} \\
\text { gonorrhoeae }\end{array}$ & III & $\begin{array}{l}\text { - Silver nitrate } 1 \% \text { eye drops ( }(n) \\
\text { - Erythromycin } 0.5 \% \text { ointment }(n l) \\
\text { - Tetracycline } 1 \% \text { ointment }(n l)\end{array}$ & Canada & $\begin{array}{l}2002 \\
\text { (Rev. } \\
2009)\end{array}$ \\
\hline [29] & Moore W. and Nischal K.K. & $\begin{array}{l}\text { Pharmacologic } \\
\text { management of } \\
\text { glaucoma in childhood }\end{array}$ & Glaucoma & । & 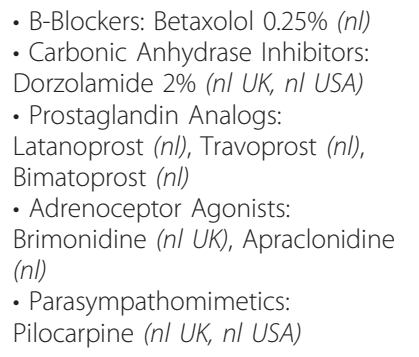 & UK & 2007 \\
\hline [39] & $\begin{array}{l}\text { Royal College of } \\
\text { Ophthalmologists }\end{array}$ & $\begin{array}{l}\text { Guidelines for the } \\
\text { management of } \\
\text { amblyopia }\end{array}$ & Ambliopia & III & $\begin{array}{l}\text { - Refractive correction (glasses) } \\
\text { - Patching: from } 2 \text { to } 6 \text { hours per } \\
\text { day } \\
\text { - Atropine ( } n / I T, n / \text { USA) }\end{array}$ & UK & 2006 \\
\hline
\end{tabular}


Table 3 Summary of guidelines on pharmacological therapy of ocular disease in the paediatric population (Continued)

\begin{tabular}{|c|c|c|c|c|c|c|c|}
\hline [6] & $\begin{array}{l}\text { National Guideline } \\
\text { Clearinghause (NGC) }\end{array}$ & $\begin{array}{l}\text { Best evidence statement } \\
\text { (BESt). Treatment of } \\
\text { amblyopia in children. }\end{array}$ & Amblyopia & I & $\begin{array}{l}\text { - Refractive correction (glasses) } \\
\text { - Atropine: } 1 \text { drop/day, } 2 \text { - } 7 \text { days } \\
\text { per week ( } n / I T, n / \text { USA) } \\
\text { - Patching: from } 2 \text { to } 6 \text { hours per } \\
\text { day }\end{array}$ & USA & 2007 \\
\hline [38] & $\begin{array}{l}\text { Royal College of } \\
\text { Ophthalmologists }\end{array}$ & $\begin{array}{l}\text { Guidelines for the } \\
\text { management of } \\
\text { strabismus in childhood }\end{array}$ & Strabismus & III & $\begin{array}{l}\text { - Surgical interventions } \\
\text { - Refractive correction (glasses) } \\
\text { - Miotics (not specified) }\end{array}$ & UK & 2007 \\
\hline [46] & $\begin{array}{l}\text { The Brazilian Society of } \\
\text { Pediatrics, Brazilian Council of } \\
\text { Ophthalmology, Brazilian } \\
\text { Society of Pediatric } \\
\text { Ophthalmology }\end{array}$ & $\begin{array}{l}\text { Brazilian guidelines } \\
\text { proposal for screening } \\
\text { and treatment of } \\
\text { retinopathy of } \\
\text { prematurity (ROP) }\end{array}$ & $\begin{array}{l}\text { Retinopathy of } \\
\text { prematurity } \\
\text { (ROP) }\end{array}$ & III & $\begin{array}{l}\text { Surgical interventions+post surgical } \\
\text { treatment with topical steroids/ } \\
\text { antibiotics (not specified) }\end{array}$ & Brazil & 2007 \\
\hline [40] & $\begin{array}{l}\text { Royal College of } \\
\text { Ophthalmologists, Royal } \\
\text { College of Paediatrics and } \\
\text { Child Health, British Association } \\
\text { of Perinatal Medicine \& BLISS }\end{array}$ & $\begin{array}{l}\text { Guideline for the } \\
\text { Screening and } \\
\text { Treatment of } \\
\text { Retinopathy of } \\
\text { Prematurity }\end{array}$ & $\begin{array}{l}\text { Retinopathy of } \\
\text { prematurity } \\
(\mathrm{ROP})\end{array}$ & III & $\begin{array}{l}\text { Screening examination with } \\
\text { Cyclopentolate } 0.5 \% \text { /Phenylephrine } \\
2.5 \% \text { combination: } 1 \text { drop each in } 2 \\
\text { to } 3 \text { doses, each } 5 \text { minutes apart, } 1 \\
\text { hour prior to examination }(n /)\end{array}$ & UK & 2008 \\
\hline
\end{tabular}

NOTE: nl: not licensed for paediatric use; IT: Italy; UK: United Kingdom; USA: United States of America

dry eye syndrome (2), and pterygia (1), and 4 RCTs on bevacizumab in the treatment of neovascular glaucoma in children $>3$ years (all 3 completed) and in ROP in neonates $>5$ months ( 1 ongoing RCT).

Among the drugs that had the most ongoing studies were also two anti-hystamine drugs, ketotifen and bepotastine, and the antibacterial moxifloxacin: these were tested in 3 RCTs each for the treatment of allergic or bacterial conjunctivitis in children.

\section{EMA/FDA viewpoint}

Although no ophthalmologic drugs are found in the EMA's priority list for studies into off-patent paediatric medicinal products at this time, the EMA Paediatric Committee (PDCO) adopted opinions on PIPs for 12 ocular medications, with the aim to generate the necessary quality, safety, and efficacy data to support the authorization of these medicines for use in children.

Four drugs, cysteamine, latanoprost, voclosporin and the recombinant human monoclonal antibody to human interleukin 17A received a go-ahead for a PIP, while one, travoprost/brinzolamide, was refused it. In four cases, one involving the anti-inflammatory agent bromfenac, one a new drug, ocriplasmin, for the treatment of symptomatic focal vitreomacular adhesion, and two the vascular endothelial growth factor inhibitors, ranimizumab and pegaptanib, a waiver was granted in all age groups on the grounds that the specific medicinal product does not represent a significant therapeutic benefit or because the disease or condition for which the product is intended does not occur in the specified paediatric subset(s). Finally, 2 steroid drugs, dexamethasone and triamcinolone, were refused the granting of a product-specific waiver on the grounds that the clinical studies cannot fulfil a therapeutic need of the paediatric population.

By consulting the "List of the active substances included in the work-sharing procedure in accordance with Articles 45 and 46 of the European Paediatric Regulation, no additional data or information on their use in the paediatric population resulted to be submitted or requested to authorise the paediatric use of any ocular medicinal product.

Twenty-six ocular medications were found in the Food and Drug Administration (FDA)'s "Table of Medicines with New Paediatric Information", a list of drugs approved for use in the paediatric population resulting from the paediatric clinical trials performed in response to paediatric legislative initiatives. Ten (38\%) were anti-allergy medications, 8 (31\%) were anti-glaucoma medications (6 of which were not yet licensed for paediatric use in the USA), and 5 were antibacterials and combinations. The last three agents were triamcinolone (steroid agent), lidocaine (local anaesthetic agent), and a hypromellose combination (lubricant). These drugs included approved information on use in the paediatric population resulting from the paediatric clinical trials performed in response to paediatric legislative initiatives.

\section{Discussion}

This article reviews ocular medication use in children, providing a summary of their licensing status in Italy, the UK, and the USA and analyse the amount of available studies testing these medicines in the paediatric population. Most of the drugs listed have only recently obtained paediatric use approval and are now widely prescribed for children by a growing number of clinicians [29]. However, for most of these drugs wide differences in the licensed age groups were found and only a few are available in all three countries. Even if the Paediatric Regulation in EU and USA specifically aims at giving children the same access to authorised medicinal products suitable for their use, the age approval and occasionally the approach towards certain therapeutic problems is under 
the direct responsibility of National Authorities, so differences in drug licensing procedure between countries remain. There is therefore a need for evidence-based harmonization of drug licenses in order to guarantee equal drug availability and access [30].

Furthermore, many ocular medications commonly used in children still do not have paediatric dosing and safety labelling information in any country. For example, almost for all glaucoma medications (such as prostaglandin analogues and carbonic anhydrase inhibitors), paediatric use is labelled "not recommended".

At this time no paediatric RCTs were available for several ocular medications. When available, the studies were often limited to small case series and case reports, so more extensive controlled trials will be needed to confirm their safety and efficacy also in paediatric population. On the contrary, evidence on efficacy was found for drugs that were not licensed for children, such as tetracycline and bupivacaine.

In spite of the fact that no ophthalmologic drugs are found in the EMA's priority list, several drugs were recently studied in paediatric clinical trials in the European countries and the USA. In particular, the ongoing research is examining the potential use of intravitreally injected anti-VEGF drugs, such as bevacizumab, successfully used in adults with diabetic retinopathy or age-related macular degeneration (AMD), a cause of a severe vision loss among the aging population in many western countries [31,32]. These drugs could now also be used in paediatric vitreoretinal diseases, as shown by recent studies on intravitreal injection of bevacizumab for the treatment of ROP, the leading cause of childhood blindness [33-37].

Moreover, the available guidelines on the pharmacological management of eye diseases in the paediatric population often recommend the use of medications not licensed or investigated in children, especially for the management of glaucoma (such as prostagliandin analogs) or acute bacterial conjunctivitis (such as steroids and antibiotics combinations). An effort to stimulate research and clinical development is therefore needed also for them, in order to guarantee medicines that have been proven to be of benefit also in paediatric patients.

Many good ethical and economical reasons exist for limiting paediatric clinical trials, while guaranteeing appropriate conclusions. Because of the characteristics of the paediatric population, limited information is also available regarding the side effects related to ocular medication use in children [38]. As the number and variety of ocular medications has increased and the number of clinicians involved in their prescription has grown, the risk of systemic adverse reactions may also increase $[39,40]$. When prescribing ocular medications in children, physicians should therefore carefully consider their risk/benefit profile, referring to details of labelling for paediatric use, such as the age of the child for whom the drug is approved, and be aware of their potentially serious systemic side effects [5].

Some strategies for reducing systemic absorption and toxicity should be followed whenever possible. First of all, the lowest available dosage of medication necessary to achieve a therapeutic benefit while minimizing risk should be used. Secondly, since different formulations may have different degrees of systemic absorption, formulations with lower systemic absorption, which may be more suitable for use in children, should be used. Ophthalmic gel or ointment, for example, has been found to have reduced systemic absorption compared to the ophthalmic solution [28]. In addition, paediatric patients should be monitored closely during and after treatment for local and systemic side effects [29].

The present findings suggest that access to, and rational use of, ocular medications in the paediatric population continue to present a considerable challenge. Paediatric clinical trials are important for defining how infants and children respond to medications and for identifying age-specific toxic effects [41]. While recent legal and economic incentives in both Europe and the USA stimulate research to obtain more data regarding dosing, efficacy, and safety of medicines used in children, problems remain in obtaining adequate evidence [42]. In this context, there is a pressing need for further clinical research to improve the quality, efficacy, and safety of ocular medications offered to paediatric patients. Clinical research must be carried out using appropriate methodologies (e.g. study design, sample size, randomization, and blinding) [38] also (and in particular) in the paediatric ophthalmic area, where effective up-to-date treatments, and additional research and education on use in children, remain priorities [43].

\section{Conclusion}

European and American legislation has established that children should have the same rights as adults to receive medicines that have been proven to be of benefit and that are unlikely to cause serious toxicity [44]. Even if the legislative initiatives in both Europe and the USA emphasizes the importance of large clinical trial in children, prioritizing the medicines to be studied on the basis of children's needs [45], differences between countries in drug licensing procedures, and occasionally in the approach towards certain therapeutic problems, may be quite significant [30]. A formulary containing common "paediatric" evidence-based safety and efficacy information could be a useful tool for improving the rational use of drugs in children and adolescents, harmonizing intercountry drug regulations and availability [46].

In addition, recommendations from high quality RCTs and systematic reviews, and effective knowledge translation 
strategies are essential to clinicians and policy makers in planning changes in practice that could ultimately improve patient- and system-related outcomes. All such considerations are priorities for an area, such as ophthalmic drug therapy in children, that is lacking evidence.

\section{Acknowledgements}

Dr. Filomena Fortinguerra holds an educational fellowship granted by Boerhinger Ingelheim Italia, which had no role in the design or conduct of this research.

Financial support: None

\section{Authors' contributions}

FF carried out the bibliograhical search, screened studies for inclusion, performed data extraction and analysis, and drafted the manuscript; AC provided methodological advice; MB participated in the design of the study and revised the manuscript. All authors read and approved the final manuscript.

\section{Competing interests}

The authors declare that they have no competing interests.

Received: 9 May 2011 Accepted: 20 January 2012

Published: 20 January 2012

\section{References}

1. Wallace DK, Steinkuller PG: Ocular Medications in Children. Clinical Pediatrics 1998, 37:645-652.

2. Cumberland PM, Pathai S, Rahi JS, for the Millennium Cohort Study Child Health Group: Prevalence of Eye Disease in Early Childhood and Associated Factors. Findings from the Millennium Cohort Study. Ophthalmology 2010, 117:2184-2190.

3. Roarty JD: Ophthalmologic drugs in infants and children. In Neonatal and Pediatric Pharmacology.. 3 edition. Edited by: Yaffe SJ, Aranda JV, Lippincott Williams \& Wilkins. Philadelphia, PA; 2005:340-348.

4. Chung I, Buhr V: Topical ophthalmic drugs and the pediatric patient. Optometry 2000, 71:511-518.

5. Coulter RA: Pediatric use of topical ophthalmic drugs. Optometry 2004, 75:419-429.

6. Levy Y, Zadok D: Systemic side effects of ophthalmic drops. Clin Pediatr (Phila) 2004, 43:99-101.

7. Metry DW, Hebert AA: Topical therapies and medications in the paediatric patient. Pediatr Clin North Am 2000, 47:867-876.

8. Martinez SV, Junceda MC: Systemic adverse reactions to topical ocular medications in children: A call for attention. Acta Pediátrica Española 2005, 63:121-122.

9. Coppens G, Stalmans I, Zeyen T, Casteels I: The safety and efficacy of glaucoma medication in the pediatric population. J Pediatr Ophthalmol Strabismus 2009, 46:12-18.

10. World Health Organization (WHO): Collaborating Centre for Drug Statistics Methodology. World Health Organization (WHO); [http://www.whocc.no/ atcddd/], Accessed on: 5/5/2011.

11. Elsevier: Banche dati dell'Informatore Farmaceutico. [http://www.codifa.it], Elsevier Accessed on: 5/5/2011.

12. British National Formulary 2010-2011: British National Formulary for Children. British National Formulary 2010-2011; [http://bnfc.org/bnfc/index. htm], Accessed on: 5/5/2011.

13. PDR Network: Physicians' Desk Reference ${ }^{\circledast}$ (PDR ${ }^{\oplus}$ ). [http://www.pdr.net/ Default.aspx], PDR Network Accessed on: 5/5/2011.

14. World Health Organization (WHO): WHO The International Clinical Trials Registry Platform. ICTRP. World Health Organization (WHO); [http://www. who.int/trialsearch], Accessed on: 5/5/2011.

15. US National Institutes of Health: ClinicalTrials.gov registry. US National Institutes of Health; [http://clinicaltrials.gov/ct2/search], Accessed on: 5/5/2011.

16. Current Controlled Trials Ltd: International Standard Randomised Controlled Trial Number Register. ISRCTN. Current Controlled Trials Ltd; [http://www.controlled-trials.com/isrctn/], Accessed on: 5/5/2011.
17. European Medicines Agency (EMA): Opinions and Decisions on Paediatric Investigation Plans (PIPs). European Medicines Agency (EMA); [http://www. ema.europa.eu/ema/index.jsp?curl=pages/medicines/landing/pip_search. jsp\&murl=menus/medicines/medicines.jsp\&mid=WC0b01ac058001d129], Accessed on: 5/5/2011.

18. European Medicines Agency (EMA): List of active substances for which data has been submitted in accordance with Article 45 of the Paediatric Regulation. [http://www.hma.eu/99.html], Accessed on: 5/5/2011.

19. Food and Drug Administration (FDA): Table of Medicines with New Pediatric Information. Food and Drug Administration (FDA); [http://www. fda.gov/downloads/ScienceResearch/SpecialTopics/

PediatricTherapeuticsResearc h/UCM163159.pdf], Accessed on: 5/5/2011.

20. European Medicines Agency (EMA): Revised priority list for studies into off-patent paediatric medicinal products for the Fifth Call 2010 of the 7th Framework Programme of the European Commission. European Medicines Agency (EMA); [http://www.ema.europa.eu/docs/en_GB/ document_library/Other/2009/10/WC500004017.pdf], Accessed on: 5/5/2011.

21. University of Texas, School of Nursing, Family Nurse Practitioner Program: Guidelines for the treatment and management of acute bacterial conjunctivitis in children and adults (2005). University of Texas, School of Nursing Austin (TX); [http://www.guidelines.gov/content.aspx?id=7353], Accessed on: 5/5/2011.

22. Cincinnati Children's Hospital Medical Center: Best evidence statement (BESt). Treatment of amblyopia in children. Cincinnati Children's Hospital Medical Center; 2007 [http://www.guideline.gov/content.aspx?id=14288], Accessed on: 5/5/2011.

23. The Royal College of Ophthalmologists. Paediatric Sub-Committee: Guidelines for the Management of Amblyopia. The Royal College of Ophthalmologists; 2006 [http://www.rcophth.ac.uk/page.asp? section=493\&search=], Accessed on: 5/5/2011.

24. Taylor RH: Guidelines for the Management of Strabismus in Childhood. [http://rcophth-website.www.premierithosting.com/docs/publications/paedpatient-information/Strabismus.pdf], June 2007 Author Review Date 2009. The Royal College of Ophthalmologists Accessed on: 5/5/2011.

25. Moore W, Nischal KK: Pharmacologic management of glaucoma in childhood. Paediatr Drugs 2007, 9:71-79.

26. Royal College of Paediatrics and Child Health: Guideline for the Screening and Treatment of Retinopathy of Prematurity. 2008 [http://www.rcophth. ac.uk/page.asp? section=451\&sectionTitle=Clinical+Guidelines], Accessed on: $5 / 5 / 2011$.

27. Zin A, Florêncio T, Fortes Filho JB, Nakanami CR, Gianini N, Graziano RM, et al: Brazilian guidelines proposal for screening and treatment of retinopathy of prematurity (ROP). Ara Bras Oftalmol 2007, 70:875-883.

28. Canadian Paediatric Society, Infectious Diseases and Immunization Committee: Recommendations for prevention of neonatal ophthalmia. Can Med Assoc J 2002, 129:554-555.

29. Gray C: Systemic toxicity with topical ophthalmic medications in Children. Paed Perinat Drug Ther 2006, 7:23-29.

30. Abraham J: Making regulation responsive to commercial interests: streamlining drug industry watchdogs. BMJ 2002, 325:1164-1169.

31. Nonobe NI, Kachi S, Kondo M, Takai Y, Takemoto K, Nakayama A, et al: Concentration of vascular endothelial growth factor in aqueous humor of eyes with advanced retinopathy of prematurity before and after intravitreal injection of bevacizumab. Retina 2009, 29:579-585.

32. Sassa $Y$, Hata $Y$ : Antiangiogenic drugs in the management of ocular diseases: Focus on antivascular endothelial growth factor. Clin Ophthalmol 2010, 4:275-283.

33. Kusaka S, Shima C, Wada K, Arahori H, Shimojyo H, Sato T, et al: Efficacy of intravitreal injection of bevacizumab for severe retinopathy of prematurity: a pilot study. $\mathrm{Br} J$ Ophthalmol 2008, 92:1450-1455.

34. Lalwani GA, Berrocal AM, Murray TG, Buch M, Cardone S, et al: Off-label use of intravitreal bevacizumab (Avastin) for salvage treatment in progressive threshold retinopathy of prematurity. Retina 2008, 28(Suppl 3):S13-18

35. Lin KL, Hirose T, Kroll AJ, Lou PL, Ryan EA: Prospects for Treatment of Pediatric Vitreoretinal Diseases with Vascular Endothelial Growth Factor Inhibition. Seminars in Ophthalmology 2009, 24:70-76.

36. Mantagos IS, Vanderveen DK, Smith LE: Emerging Treatments for Retinopathy of Prematurity. Seminars in Ophthalmology 2009, 24:82-86. 
37. Quiroz-Mercado H, Martinez-Castellanos MA, Hernandez-Rojas ML, SalazarTeran N, Chan RV: Antiangiogenic therapy with intravitreal bevacizumab for retinopathy of prematurity. Retina 2008, 28(Suppl 3):S19-25.

38. Macrae D: Conducting clinical trials in pediatrics. Crit Care Med 2009, 37(Suppl 1):S136-139.

39. Labetoulle M, Frau E, Le Jeunne C: Systemic adverse effects of topical ocular treatments. Presse Med 2005, 34:589-595.

40. Sheldrick JH, Wilson AD, Vernon SA, Sheldrick CM: Management of ophthalmic disease in general practice. Br J Gen Pract 1993, 43:459-462.

41. Hoppu K: Paediatric clinical pharmacology at the beginning of a new era. Eur J Clin Pharmacol 2008, 64:201-205.

42. Jefferys $\mathrm{DB}$, Jones $\mathrm{KH}$ : Emea and the new pharmaceutical procedures for Europe. Eur J Clin Pharmacol 1995, 47:471-476.

43. Pandolfini C, Bonati M: European paediatric research and children's therapeutic needs. Acta Pardiatrica 2008, 97:1232-1237.

44. Choonara I: Regulation of drugs for children in Europe. BMJ 2007, 335:1221-1222.

45. Hoppu K: Can we get the necessary clinical trials in children and avoid the unnecessary ones? Eur J Clin Pharmacol 2009, 65:747-748.

46. Bonati $\mathrm{M}$, Pandolfini : Is it time for a European formulary of paediatric medicines? Arch of Dis Child 2004, 89:890-891.

\section{Pre-publication history}

The pre-publication history for this paper can be accessed here: http://www.biomedcentral.com/1471-2431/12/8/prepub

doi:10.1186/1471-2431-12-8

Cite this article as: Fortinguerra et al:: Ocular medicines in children: the regulatory situation related to clinical research. BMC Pediatrics 2012 12:8.

\section{Submit your next manuscript to BioMed Central and take full advantage of:}

- Convenient online submission

- Thorough peer review

- No space constraints or color figure charges

- Immediate publication on acceptance

- Inclusion in PubMed, CAS, Scopus and Google Scholar

- Research which is freely available for redistribution

Submit your manuscript at www.biomedcentral.com/submit 